\title{
CAN MOTION ON A TRACK MIMIC A FORCED, DAMPED NONLINEAR OSCILLATOR?
}

\section{H.P.W. GOTTLIEB}

School of Science, Griffith University,

Nathan, Brisbane, Queensland, Australia 4111

(January 2002)

Running title: OSCILLATOR MIMICRY AND MOTION ON A TRACK

Number of pages: 8

[Number of Figures: 0] 


\section{INTRODUCTION}

In some earlier papers ([1] - [3]) the author considered the correspondence between frictionless motion of a particle on a curved track under the influence of gravity and one-dimensional oscillator motion due to a potential. Systems for which the motion on a track, projected onto the horizontal axis, was exactly the same as a one-dimensional motion due to a potential were termed isodynamical. The potential isodynamical to any given track shape was found in [1]; and the tracks isodynamical to any given potential were treated in [2].

The above work dealt with undamped (frictionless) and unforced (undriven) motion. Insofar as forced oscillations exhibit a much richer range of phenomena, it is therefore of interest to know further whether a track could be constructed, for which the projected motion is isodynamical to a forced, possibly damped, nonlinear oscillator. That is, can a mechanical system still exactly mimic the one-dimensional oscillator dynamics?

Gottwald et al. [4] attempted an experimental track realization of the Duffing equation, but they made approximations, and actually dealt with mimicry of general phenomena rather than exact mimicry (isodynamism) of the oscillator equation. At about the same time, Shaw and Haddow [5] investigated the dynamics of "rollercoaster" experimental models as analogue devices for nonlinear oscillators, including the effects of friction and excitation by horizontal support motion. As emphasized by them, they chose the arc-length coordinate equation as the dynamical equation required to have the same form as the oscillator equation. Their example dealing with 
the forced situation also involved some approximations. In a recent book, Virgin [6] has extended these investigations.

If friction is included, then a common qualitative way of thinking (e.g. Alligood et al. [7, p.378]) is to include a linear damping term in an oscillator equation, and imagine a ball rolling up and down a potential well of the same shape as obtained from the energy equation and losing energy because of friction. However, this correspondence is not expected to be exact.

In this paper, we attempt to include (viscous) friction, proportional to velocity, and driven motion. The differential equations for all these situations are derived. It is shown that isodynamical motion, as we have defined it above, is not possible when the damping is included; nor is it possible for motion forced by a horizontally driven, oscillating track. 


\section{DAMPED MOTION}

\subsection{DAMPED, NONLINEAR OSCILLATOR}

For a particle, with mass $\mathrm{m}$, whose motion in one dimension is due to a potential $\mathrm{v}(\mathrm{x})$ in the presence of viscous damping, with coefficient $\gamma$, the equation of motion is

$$
m \ddot{x}+\gamma \dot{x}+d v / d x=0 .
$$

Using $\ddot{x}=(1 / 2)(d / d x)\left(\dot{x}^{2}\right)$ and $d x=\dot{x} d t$, this may be integrated to give

$$
\dot{x}^{2}+(2 / m) v(x)=-2(\gamma / m) \int \dot{x}^{2} d t .
$$

\subsection{MOTION ON A TRACK WITH FRICTION}

A particle, say a bead, with mass M, slides, under the influence of gravity (constant g), along a track which lies in a vertical plane and which has equation $\mathrm{Y}=$ $\mathrm{Y}(\mathrm{X})$, where $\mathrm{X}$ is the horizontal coordinate and $\mathrm{Y}$ the vertical coordinate. If the friction were of the "dry" type, proportional to the normal reaction, then the equations of motion would not contain a velocity-type term such as that in the usual damped oscillator equation (2.1). Therefore, for the possibility of motion isodynamical with the damped oscillator, the friction associated with motion along the track must evidently be of the "viscous" type, proportional to tangential velocity. The friction forces in the $\mathrm{X}$ and $\mathrm{Y}$ directions are then simply $-\Gamma \dot{X}$ and $-\Gamma \dot{Y}$ respectively $(\Gamma$ constant). 
The vertical acceleration can be expressed in terms involving the slope $Y^{\prime}$ by

$$
\ddot{Y} \equiv Y^{\prime} \ddot{X}+Y^{\prime \prime}(\dot{X})^{2}
$$

Resolution of forces and elimination of the normal reaction leads eventually to the equation for motion projected onto the horizontal axis:

$$
\ddot{X}=-\left\lfloor\left(g+Y^{\prime \prime} \dot{X}^{2}+(\Gamma / M) Y^{\prime} \dot{X}\right) Y^{\prime}+(\Gamma / M) \dot{X}\right\rfloor /\left\lfloor 1+\left(Y^{\prime}\right)^{2}\right\rfloor .
$$

(C.f. equation (2.3) in ref. [1] when $\Gamma=0$.) This can be integrated to give

$$
\left.\dot{X}^{2}+2 g Y /\left[1+\left(Y^{\prime}\right)^{2}\right]=-2(\Gamma / M) \mid \int \dot{X}^{2}\left[1+\left(Y^{\prime}\right)^{2}\right] d t\right] /\left[1+\left(Y^{\prime}\right)^{2}\right]
$$

Comparison of the right-hand side of equation (2.2) with that of (2.5) shows that they cannot be of similar form, except for the trivial case of constant slope. (Actually, piecewise constant slope, such as a V-shape, could be accommodated - c.f. the Appendix of ref. [1] for the undamped case - but this will not be considered further here as it is not a smooth shape.)

It is thus concluded that, unlike the undamped case, no isodynamical systems of potential motion and curved track can be found when friction is present. 


\section{FORCED MOTION}

\subsection{FORCED NONLINEAR OSCILLATOR}

The equation of motion in one dimension for a particle of mass $m$ due to a potential $\mathrm{v}(\mathrm{x})$ and subject to a driving force written as $m \hat{d}(t)$ is

$$
m \ddot{x}+d v / d x=m \hat{d}(t),
$$

which may be integrated once to give

$$
\dot{x}^{2}+(2 / m) v=2 \int \dot{x} \hat{d}(t) d t .
$$

\subsection{MOTION ON A DRIVEN TRACK}

The track (regarded as a rigid shape) may be subjected to a prescribed horizontal displacement, i.e. shaken, as in ref. [4] for harmonic excitation or ref.[5] more generally. (In view of the result of Section 2 above, the motion is taken to be undamped.) The track shape is now denoted by $\mathrm{F}(\mathrm{X})$, and the track undergoes the given time-dependent displacement $\mathrm{D}(\mathrm{t})$. At time $\mathrm{t}$, the vertical coordinate of the particle (bead of mass M) is therefore given by

$$
Y(X)=F[\Xi(t)] \quad ; \quad \Xi(t) \equiv X-D(t) .
$$

The slope at $X$ is therefore given by $F^{\prime}$, where the prime now denotes differentiation with respect to $\Xi$. 
For the possibility of isodynamical motion, a horizontal coordinate change of independent variable is made, from $X$ to $\Xi$, so that, when $\mathrm{F}=0$, the equation for $\ddot{\Xi}$ looks like the equation for $\ddot{x}$ when $\mathrm{v}=0$. The equation of motion relative to the moving frame, using equation (3.3), may then be shown to be

$$
\ddot{\Xi}=-\left\lfloor\left(g+F^{\prime \prime} \dot{\Xi}^{2}\right) F^{\prime}+\ddot{D}\right\rfloor /\left[1+\left(F^{\prime}\right)^{2}\right\rfloor .
$$

(When $\mathrm{D}=0$, this agrees with equation (2.3) of ref.[1]. For D nonzero and $\mathrm{F}=0$, this can look like equation (3.1) with $v=0$.) Equation (3.4) can be integrated once to give

$$
\dot{\Xi}^{2}+\frac{2 g F(\Xi)}{1+\left(F^{\prime}\right)^{2}}=\frac{2 \int \dot{\Xi} \ddot{D} d t}{1+\left(F^{\prime}\right)^{2}} \text {. }
$$

Comparison with the right-hand side of equation (3.2) shows that, once again, because the denominator in the last term of equation (3.5) is a function of coordinate, this projected motion (relative to the driven track frame) cannot be isodynamical with an oscillator in the forced case.

\section{ACKNOWLEDGMENTS}

The author thanks the Department of Mathematics at the University of Queensland for hospitality during an Academic Studies Program visit there. 


\section{REFERENCES}

1. H. P. W. GOTTLIEB 1997 Journal of Sound and Vibration 199, 667-678. Isodynamical tracks and potentials. doi:10.1006/jsvi.1996.0659

2. H. P. W. GOTTLIEB 1997 Journal of Sound and Vibration 204, 519-532. Exact mimicry of nonlinear oscillatory potential motion: Nonuniqueness of isodynamical tracks. doi:10.1006/jsvi.1997.0957

3. H. P. W. GOTTLIEB 1997 Nonlinear Analysis, Theory, Methods \& Applications 30, 5157-5165. Isodynamical systems.

4. J. A. GOTTWALD, L. N. VIRGIN and E. H. DOWELL 1992 Journal of Sound and Vibration 158, 447-467. Experimental mimicry of Duffing's equation.

5. S. W. SHAW and A. G. HADDOW 1992 Nonlinear Dynamics 3, 375-384. On 'roller-coaster' experiments for nonlinear oscillators.

6. L. N. VIRGIN 2000 Introduction to Experimental Nonlinear Dynamics. Cambridge: Cambridge University Press.

7. K. T. ALLIGOOD, T. D. SAUER and J. A. YORKE 1997 Chaos. New York: Springer. 